AperTO - Archivio Istituzionale Open Access dell'Università di Torino

An update on the treatment of cytomegalovirus infection after allogeneic hematopoietic stem cell transplantation

This is a pre print version of the following article:

Original Citation:

Availability:

This version is available http://hdl.handle.net/2318/1725742

since 2020-01-31T16:40:44Z

Published version:

DOI:10.1080/17474086.2019.1657399

Terms of use:

Open Access

Anyone can freely access the full text of works made available as "Open Access". Works made available under a Creative Commons license can be used according to the terms and conditions of said license. Use of all other works requires consent of the right holder (author or publisher) if not exempted from copyright protection by the applicable law. 


\title{
An Update on the Treatment of CMV Infection after Allogeneic Hematopoietic Stem Cell Transplantation.
}

\author{
Maffini E, ${ }^{1,2}$ Busca A, ${ }^{1}$ Costa $\mathbf{C},{ }^{3}$ Giaccone L, ${ }^{1,2}$ Cerrano M, ${ }^{1,2}$ Curtoni A, ${ }^{3}$ Cavallo $\mathbf{R},{ }^{3,4}$ \\ Bruno B ${ }^{1,2}$ \\ ${ }^{1}$ Department of Oncology, SSCVD Trapianto di Cellule Staminali, A.O.U. Città della Salute e della Scienza \\ di Torino, Torino, Italy; ${ }^{2}$ Department of Molecular Biotechnology and Health Sciences, University of \\ Torino, Torino, Italy; ${ }^{3}$ SC Microbiology and Virology, A.O.U. Città della Salute e della Scienza di Torino, \\ Torino, Italy; ${ }^{4}$ Department of Public Health and Pediatrics, University of Torino, Torino, Italy.
}

Corresponding Author: Enrico Maffini, MD

\begin{abstract}
:
Human Cytomegalovirus (CMV) remains a major cause of morbidity and mortality after allogeneic hematopoietic stem cell transplantation (allo-HSCT). Frequency of CMV infection and disease after allo-HSCT depend on several factors including virus-host interactions, recipient-donor CMV serology, donor type (HLA-identical vs. haplo-identical donors)and intensity of conditioning regimens. Moreover, monitoring strategies, cut-off values of viremia to guide prophylactic, preemptive, and treatment interventions remain to be determined. Broader knowledge in CMV biology and its relationship with the host immune-system is greatly contributing to develop novel therapeutic approaches including the application of novel antiviral drugs, with improved toxicity profiles currently under investigation in randomized phase II and III trials, and cell therapy approaches. Significant evidence of the clinical efficacy of adoptive T-cell therapy (ATCT) from CMV-seropositive donors is also emerging. Alternative methods allow ATCT also in allo-HSCT recipients of seronegative donors and cord blood grafts. Vaccine-immunotherapy has the difficult task to reduce the incidence of CMV reactivation/infection in immunocompromised allo-HSCT patients and reach CMV immune control. In this manuscript, we update a previous review by also including recent developments in the virology lab and their possible association with treatment strategies at bedside.
\end{abstract}


Key words: cytomegalovirus (CMV), immune response, CMV-vaccine, hematopoietic stem cell transplantation (HSCT), adoptive T-cell therapy (ATCT).CMV infection, CMV viral load monitoring, immune monitoring, antiviral drugs 


\section{Introduction}

Despite remarkable improvements in diagnostic tests and treatments, management of CMV in HSCT recipients remains challenging. Standard treatment options for cytomegalovirus (CMV) reactivation/infection have for long been limited to a small number of effective drugs, most of them characterized by significant toxicities, high costs and the risk of promoting drug-resistant CMV strains. Ganciclovir (GCV) and its oral bio-available pro-drug valganciclovir remain the most widely used drugs in the setting of pre-emptive treatment. Although GCV lowered CMV reactivation in previous randomized controlled clinical trials, it failed to improve overall survival rates versus placebo in CMV prophylaxis among allogeneic hematopoietic stem cell transplant (allo-HCT) recipients primarily due to myelosuppressive effects resulting in clinically relevant multiple bacterial and fungalinfections ${ }^{1-3}$. Foscarnet, a virustatic DNA-polymerase inhibitor, represents an alternative to GCV due to the absence of myelotoxicity but its nephrotoxic effect coupled with marked serum electrolytes abnormalities, requires a very cautious approach, rendering its management potentially difficult ${ }^{4}$. The emergence of drug-resistant CMV strains poses a continuous major threat to HSCT recipients, with relevant rates of morbidity, graft losses and, mortality. With the recent introduction of novel agents such letermovir, prevention of CMV infection in allo-HCT recipients may gradually change the clinical approach shifting the paradigm from preemptive strategies to effective prophylaxis. Moreover, the emerging role of cell-mediated immunologic monitoring and CMV specific viralogical testing may be of great help in clinical decision-making process. In this review, we also discuss the integration of novel antiviral therapies, including CMV specific $\mathrm{T}$ cell therapies, with standardized molecular tests that may lead to individualized prophylactic and therapeutical approaches.

\section{CMV infection and replication in human cells}

CMV is a member of the family Herpesviridae, responsible for asymptomatic and generally benign self-limiting infections in immunocompetent subjects. CMV genome consists of $230 \mathrm{kbp}$ linear double-stranded DNA encoding structural and functional proteins, for instance UL97 and UL54 that are extremely relevant in the context of anti-CMV agents. CMV is able to replicate in epithelial cells, fibroblasts and monocytes/macrophages. Once the CMV penetrate into the infected cell the viral DNA is transported into the nucleus for transcription. At this point, early genes initiate the replication of viral genome. CMV encodes for its own DNA polymerase and the result of CMV replication is a long DNA chain containing multiple repeated gene sequences known as concatemers. The production of infective virions requires the cleavage of the concatemeric DNA 
into multiple monomers that will be eventually packaged into a viral capside prior to its release as an infective virion. These steps of maturation, packaging and termination are performed by a group of proteins defined as the "CMV terminase complex" including the pUL9 and pUL56 that interact synergistically. Once the terminase complex has cleaved the CMV concatemers, the CMV DNA is transported inside an empty viral procapsid by the interaction of the terminase complex and capsid proteins and finally the enveloped infectious particles are released. Of note, the structure of terminase complex is conserved across members of herperviridiae but it is not shared with human cells: accordingly, agents interacting with the terminase complex might be potentially virus-specific while preserving human cells.

\section{CMV infection and disease after allo-HSCT: the role of the virological evaluation}

The frequency of CMV infection and disease after allo-HSCT relies on the interaction of several factors pertinent to the virus, the host, and the type of transplant. ${ }^{6,7}$ Among virus-related factors, the development of CMV disease has directly been correlated with viral replication and related CMV DNAemia. Considering the antiviral strategies to prevent CMV disease, prophylaxis and pre-emptive therapy are commonly recognized as current standard approaches. ${ }^{8-12} \mathrm{~A}$ recent systematic meta-analysis on the role of prophylaxis in HSCT and several controlled trials on the efficacy of different protocols for CMV prophylaxis have clearly showed the formidable role played by specific antiviral prophylaxis and pre-emptive therapies. ${ }^{13}$ In this setting, virological testing is pivotal. Close viral monitoring and initiation of antiviral treatment at a certain threshold of CMV DNAemia are crucial for effective pre-emptive strategies. In the past decades, an antigenemia assay, that allowed quantitative detection of CMV immediate early antigens pp65-UL83 in peripheral polymorphonuclear cells, played a key role in monitoring CMV reactivation and infections in both solid organ and allo-HSCT recipients and in guiding pre-emptive treatments. However, this assay has gradually become obsolete as it was labor- and time consuming, and highly operator-dependent. Presently, molecular methods such as commercial available, fully automated PCR assays represent the gold standard given their standardization. These technologies are completed by nucleic acid extraction platforms, implemented to extract and quantify CMV DNA from either plasma or whole blood specimens. Importantly, it is generally assumed that plasma CMV DNAemia is about $1-\log 10$ lower than whole blood DNAemia. Moreover, a recent Italian retrospective non-interventional multicenter cohort study described different kinetics of CMV DNA in blood and plasma with prolonged persistence of viral DNA in plasma rather than whole blood following ganciclovir treatment, despite adequate control of viral replication. ${ }^{14}$ These findings suggest that viral 
monitoring on plasma samples may lead to an inappropriately prolonged antiviral treatment with inevitable increased risk of toxicity. Overall, whole blood DNAemia may therefore be preferred to guide pre-emptive treatment. It is at least strongly recommended to use the same biological fluid throughout the patient follow up given the significantly different CMV DNAemia levels in blood and plasma. $^{15}$

Some issues that link virological testing with clinical approaches remain to be addressed. No definitive threshold of viral load for starting pre-emptive treatment has been determined. Some Authors have suggested that pre-emptive treatment should be started for levels of CMV-DNAemia> 10,000 copies $/ \mathrm{mL}$ in whole blood or $>1000$ copies $/ \mathrm{mL}$ in plasma. A lower threshold could be considered in presence of high risk of viral disease (i.e. early infection within the first month posttransplant, cord blood recipients, active anti-GVHD treatment after a transplant from haploidentical or mismatched unrelated donor, positive donor CMV serology and recipient negative, exvivo or in-vivo T cell depletion). Considering viral kinetics, at least a threefold increase in viral load should be considered significant. When the cut-off threshold is reached, CMV DNAemia should be confirmed within a couple of days. Regardless of its duration, pre-emptive therapy should be discontinued in case of CMV-DNAemia clearance after two consecutive negative tests performed at least 3-4 days apart. This is particularly relevant to reduce the risk of selecting drug resistant strains. Another technical but relevant issue is the high variability of molecular assays for low CMV DNAemia loads $(<1000 \mathrm{IU} / \mathrm{mL})$. It may be reasonable to think that changes greater than threefold $\left(0.5 \log _{10} \mathrm{IU} / \mathrm{mL}\right)$ represent biologically important variations in viral replication with potential clinical impact. ${ }^{16}$ Of note, when interpreting CMV DNAemia levels, close attention should be paid to the mechanism of action of the antiviral agent used for prophylaxis/pre-emptive therapy. In fact, while acyclovir, ganciclovir, valganciclovir and foscarnet inhibit viral replication by interfering with CMV DNA polymerase function, the more recently developed letermovir does not interfere with DNA synthesis but inhibits the terminase complex subunit pUL56 at a viral maturation and packaging stage of viral particles subsequent to viral DNA synthesis. ${ }^{17,18}$ Therefore, CMV DNAemia may remain longer detectable in patients receiving letermovir. ${ }^{12}$ Although this could be less relevant during prophylaxis, breakthrough CMV DNAemia in patients receiving prophylaxis with letermovir might be of difficult interpretation. Importantly, in 2010, the World Health Organization (WHO) International Reference Standard for CMV-DNA quantification was developed and approved. This allowed for the calibration of CMV-specific quantitative assays and for reporting as IU/mL, overall improving inter-assay reproducibility between laboratories. ${ }^{19}$ 
Among the host-related factors, pre-transplant CMV serostatus is considered the single most important predictor of CMV disease post-transplant with seronegative recipients from a seropositive donor ( $\mathrm{D}+/ \mathrm{R}-)$ being at the highest risk; seropositive recipients $(\mathrm{D}+/ \mathrm{R}+; \mathrm{D}-/ \mathrm{R}+)$ at intermediate risk, and D-/R- at the lowest risk ${ }^{20-22}$ According to a well-recognised clinical practice, pre-transplant serological evaluation for presence / absence of anti-CMV IgG must invariably be performed in both donor and recipient. Ifthe serological status is negative in donor and/or recipient, a reevaluation at the time of transplant should be recommended. If the recipient is seronegative, a seronegative donor should be selected when possible. Moreover, if CMV specific IgMs are detected, the viral load should be evaluated by molecular methods and, in the presence of CMV DNAemia, the candidate donor should temporarily be excluded from donation.

\section{CMV infection and CMV-specific T cell response}

An important predictor of CMV infection/disease is represented by CMV-specific $\mathrm{T}$ cell response. $^{23,24}$ Different methods to measure cellular immune response are available, including flow cytometry-based techniques and interferon gamma releasing assays (IGRAs). IGRAs have been developed to detect and quantify $\mathrm{T}$-cell responses following antigen-specific stimulation and to give an indication of functional responsiveness. ${ }^{25-30}$ QuantiFERON-CMV is the only IGRA assay approved for CMV immune monitoring in the clinical setting. It consists in the quantification, by immuno-enzymatic technique, of the patient CMV-specific CD8+ Tcells following ex-vivo stimulation with CMV specific peptides and evaluation of IFN- $\gamma$ release. QuantiFERON is simple and reproducible and has been validated in both SOT and allo-HSCT recipients. Another commonly employed IGRA is the CMV enzyme-linked immunospot (EliSPOT) assay for simultaneous detection and quantitation of both CD4+ and CD8+ T-cell responses. In the setting of allo-HSCT, evaluation of cellular immune response should be evaluated post-transplant when recipient lymphocytes against CMV have presumably been ablated and after engraftment when conditioninginduced lymphopenia has been resolved. The threshold of $\mathrm{CD} 3+\mathrm{T}$ cells number required for reliable testing has not yet been defined although some centers consider $100 \mathrm{~T}$ cells / $\mathrm{ul}$ as the minimum cut-off. In most studies on allo-HSCT patients, IGRAs were regularly performed (most commonly weekly or monthly) after engraftment for a variable period (during the first 100 days or the first 12 months post-transplant). ${ }^{25-30}$ In the presence of CMV-specific T-cell response in clinically stable recipients, no further evaluation is recommended. In recent years, numerous studies have assessed the role of T-cell immunological monitoring to determine the risk of CMV disease after solid organ and allo-HSCT. ${ }^{31-37}$ In both settings, after documenting CMV-specific T-cell responses, observation in the wait of a spontaneous viral clearance, with no or only short term 
administration of any antiviral treatment, could be considered. However, if poor or absent CMVspecific T-cell responses are observed, more intensive viral monitoring and more aggressive antiviral prophylaxis or therapy strategies may be considered. The impact of CMV-specific immune reconstitution was variably associated with lower incidence of CMV infection/disease, lower rate of infection recurrence, higher spontaneous viral clearance, lower peak viral loads, or shorter treatment duration. $^{25-30}$

\section{Role of recent agents}

Letermovir (LMV) - LMVbelongs to a new class of anti-CMV drugs. It inhibits the viral terminase complex by targeting the subunit UL56. Its antiviral activity is highly specific for CMV and lacks inhibitory activity against other viruses such as HSV, EBV, HZV, HHV6 and adenovirus. Interestingly, no cross-resistance has been reported between LMVand other agents including ganciclovir, foscarnet or cidofovir. In addition, based on the data currently available, primary resistance to LMVappears a rare event ${ }^{17}$.

Pharmacokinetics LMV is available as oral or intravenous formulation. It is a weak to moderate inhibitor of CYP3A resulting in increased levels of sirolimus, tacrolimus and amiodarone; a weak to moderate inducer of CYP2C9/19 resulting in decreased levels of voriconazole and PPI, and an inhibitor of OATP1B1/3 (organic anion transporting polypeptide). LMV has a remarkable interaction with cyclosporine (CSA), whose plasmatic levels may be increased during treatment. CSA is catalyzed by CYP3A4 and the effects of LMVon CSA may be explained by the inhibition of intestinal and hepatic CYP3A4 and/or P-gp. In addition, CSA may inhibit liver transporters such as ATP-binding cassette transporters resulting in increased levels of letermovir: according to this observation, the dose of LMVmust be halved when CSA is co-administered. Table 1 summarizes the relevant interactions between LMVand the agents most frequently used in hematology.

\section{Clinical Trials}

A pivotal phase II study was conducted in CMV seropositive recipients of allo-HSCT from matched related and unrelated donors. ${ }^{38}$ Patients were randomized to receive either CMV prophylaxis with LMVat three different doses (60 mg-120 mg-240 mg QD) or placebo for 12 weeks after engraftment. All-cause prophylaxis failure was defined as detectable CMV DNAemia and/or CMV disease or drug discontinuation prior to day 84 due to any reason. CMV prophylaxis failure rate was significantly higher in patients who received placebo (64\%) as compared to those who received LMVat the dose of $120 \mathrm{mg}(32 \%)$ and $240 \mathrm{mg}(29 \%)$ (p 0.01 and 0.007 respectively). Of note, in a 
post-hoc analysis, no cases of "virological failure" were observed in patients who received LMVat $240 \mathrm{mg}$ after excluding patients with detectable CMV DNAemia at baseline, as compared to $24 \%$ of the patients in the placebo group. No hematological toxicity or nephrotoxicity were reported with the administration of LMV.

Based on the encouraging results of this study, a phase III double-blind, placebo-controlled trial was designed to assess the efficacy of prophylaxis with LMVat the dose of $480 \mathrm{mg}$ QD (240 mg QD with concomitant cyclosporine administration) in adult CMV seropositive patients undergoing allo$\mathrm{HSCT}^{18}$. Patients received LMVor placebo within a median of 9 days after the graft and for up to 14 weeks after transplantation. The primary end point was the proportion of patients with clinically significant CMV infection, defined as CMV DNAemia leading to preemptive therapy or CMV disease. Overall, fewer patients in the LMV group than in the placebo group had clinically significant CMV infection by week 24 (37\% vs 61\%, p<0.001). CMV disease was observed in $1.5 \%$ of patients in the LMVgroup and $1.8 \%$ in those of the placebo group. The gastrointestinal tract was involved in all cases. Of note, the beneficial effect of LMVwas more evident in the high-risk group defined as patients grafted from mismatched related, unrelated or haplo-identical donors, patients who received cord blood or T-cell depleted transplants and patients with grade IIgraft-vs.-host disease (GVHD). The frequency of severe adverse effects was similar in the two groups.

Brincidofovir $(\boldsymbol{B D F})$ - recently published results from the randomized double-blind, placebo-controlled Phase III clinical trial (SUPPRESS) of oral BDF for CMV-seropositive alloHCT patients were disappointing ${ }^{39}$. At the dosage of $100 \mathrm{mg}$ twice weekly, the drug did not decrease CMV disease/reactivation requiring pre-emptive therapy through week 24 as compared to placebo group. BDF-associated diarrhoea - already observed in the phase 2 trial CMX001-201 could not always be differentiated from gastrointestinal (GI) manifestations of GVHD. This resulted in more frequent immunosuppressive treatments for presumed GI manifestations of grade III acute GVHD that may partly have contributed to higher rates of non-relapse mortality. As the Authors stated, a new intra-vascular formulation of BDF is warranted for a new accurate examination of its efficacy and tolerability among transplant recipients.

Maribavir (MRB)-MRB inhibits viral UL97 kinase. It was employed at the dose of $100 \mathrm{mg}$ in a multicenter, randomized, double blind, phase III study on CMV prophylaxis in allo-HSCT recipients which failed to meet the study endpoints. ${ }^{40}$ It was recently proved to be effective at much higher doses of $400 \mathrm{mg} / 800 \mathrm{mg} / 1200 \mathrm{mg}$ twice daily for resistant/refractory CMV infection in a recent randomized trial. ${ }^{41}$ In the light of these results, MRB obtained the breakthrough status designation by the Food and Drug Administration. Drop-out rate secondary to adverse events 
washowever quite high at 34\%. Of note, 13 patients developed mutations conferring MRB resistance. Two prospective trials are currently ongoing. The first (NCT02931539) will evaluate MRB $400 \mathrm{mg}$ twice daily for eight weeks in patients with resistant/refractory CMV infections. CMV viremia clearance is the primary study endpoint. The second (NCT02927067)will compare efficacy and safety of MRB at $400 \mathrm{mg}$ to valganciclovir at $900 \mathrm{mg}$ in terms of CMV viremia clearance in asymptomatic transplant recipients.

\section{CMV vaccination}

Though there is no currently licensed anti-CMV vaccine available, a number of vaccination strategies are under investigation. The induction of both T-cell and humoral immunity is a fundamental step toward the ideal anti-CMV vaccine. Here we include the strategies considered most advanced in the setting of allo-HSCT.

DNA-plasmid vaccines - In 2012 Kharfan-Dabaja et al. described the results from a phase II, placebo-controlled trial of a CMV DNA vaccine (TransVax) in allo-HSCT recipients. TransVax vaccine plasmids encode two CMV antigens, glycoprotein B and the phosphoprotein 65(pp65), designed to elicitboth host humoral and cellular immunity respectively. Although there was no apparent advantage in the vaccine arm as compared to the placebo group in terms of requirements for anti-CMV therapy $(\mathrm{p}=0.14)$, there was a significant trend for a reduction in CMV viremia episodes and improvement in time-to viremia events in the vaccine arm. ${ }^{12}$ However, disappointing results were reported in the randomized, double-blind, placebo-controlled phase III study HELIOS (NCT01877655) with the TransVax vaccine ASP0113 - a bivalent DNA vaccine encoding glycoprotein B antigens and pp65 - in 514 CMV-seropositive allo-HSCT recipients. Overall, the study failed to meet both primary - a composite endpoint of overall mortality and CMV end-organ disease within the first year post-transplant - and secondary - time to first CMV viremia and firstuse of antiviral therapy - end points.

Peptide vaccines - encouraging results came from CMVPepVax, an investigational peptide vaccine combined with an adjuvant, designed to control CMV infection in seropositive allo-HSCT recipients through the expansion of effector $\mathrm{T}$ cell subsets targeting pp65 CMV surface protein. ${ }^{42}$ Among patients without viremia, CMVPepVax was able to reconstitute consistent levels of pp65specific $\mathrm{T}$ cells, with enhanced the engagement of different effector $\mathrm{T}$ cell phenotypes. ${ }^{43}$ Most importantly, this peptide vaccine provided the proof of concept that allo-HSCT recipients are able to mount a vaccine-driven $\mathrm{CMV}$-specific T-cell response within an early post-transplant timeframe. 
However, since the product is HLA A*0201-restricted, its availability is limited. Overall, augment the potential anti-CMV effect of adoptively transferred donor-derive T cells, Gottlieb et al. tested the additional benefit of adjuvant CMV peptide-pulsed dendritic cell vaccination to prophylactic donor-derived CMV-peptide specific T cell infusion in four allo-HSCT recipients. All patients developed CMV specific immune reconstitution. The safety profile was acceptable with only one patient developing grade III acute GVHD. ${ }^{15}$

Vectored vaccine-Encouraging clinical findings were reported using the wild-type nonrecombinant modified vaccine Ankara (MVA) in a randomized, placebo-controlled double-blind study in 24 allo-HSCT recipients ${ }^{16}$ and then the MVA-based Triplex vaccine (encoding three CMV antigens: pp65, IE1 and IE2) in a phase I trial on healthy individuals. ${ }^{17}$ Confirmative results from the National Cancer Institute-sponsored phase II randomized, blinded and placebo-controlled multicentre trial, to evaluate the protective function of Triplex vaccine in CMV-seropositive alloHSCT recipients (NCT02506933) are eagerly awaited. The Triplex vaccine is also being tested in peripheral blood stem cell donors in a phase II trial (NCT03560752), designed to evaluate the prevention of CMV viremia in their recipients.

\section{Cytomegalovirus-specific adoptive $T$ cell therapy}

Virus-specific T cells are able to protect the host from developing overt viral disease. Direct infusion of CMV-specific $\mathrm{T}$ cells is the logical application of antiviral immunity restoration in patients lacking immune-competence. ${ }^{45}$ In the setting of allo-HSCT, the cellular product can be obtained both from the transplant donors or generated from third-party donors. The adoptive transfer of virus-specific $\mathrm{T}$ cells from the transplant donor has proven to be safe and effective in several clinical experiences. However, some limitations exist. First, the product can be obtained only if donor cells are available; second, the donor has to test seropositive for the targeted virus; third, the generation procedure is time consuming and costly; last, the final product is only patient specific.

An alternative process is to generate partially HLA- matched off-the shelf cryopreserved products from third-party donors, although strong evidence of long-term safety and in vivo persistence have only partially been demonstrated. Different selection strategies have been employed to obtain CMV-specific $\mathrm{T}$ cells products (i.e. in vitro stimulation and expansion, employment of peptide-HLA multimers, cytokine-capture techniques). A report from Lee et al. on results of a multicentre study of banked third-party virus-specific (CMV, EBV and adenovirus) $\mathrm{T}$ cells for 50 patients with refractory viral infections after allo-HSCT, showed an encouraging short- 
term antiviral response of $74 \%$ at 6-weeks, with only four later progressions in responders. ${ }^{23}$ Withers et al. treated allo-HSCT recipients with persistent/refractory CMV infections $(n=28)$, with third-party, ex-vivo expanded, partially HLA-matched (median HLA-matched antigens of the product was 2 out of 6 HLA-antigens) virus-specific T cells, at a median of 75 days post-transplant, in a multicentre phase I trial. Twelve-month overall response was $93 \%$ with a cumulative incidence of complete remission of $76 \%$. Of note, all three patients with active tissue infection were successfully treated after only one infusion. Overall, two patients developed de novo acute GVHD and one of them died from steroid-refractory grade Iv intestinal GVHD. Chronic GVHD was observed in 5 patients, in only one severe. The Authors demonstrated that viral replication control developed in the setting of terminally differentiated CD8+ effector CD45RA ${ }^{+} 62 \mathrm{~L}^{-} \mathrm{T}$ cells. ${ }^{24}$

In a phase I/II trial, investigators from Germany treated sixteen allo-HSCT recipients with refractory CMV infections with a single infusion of ex vivo streptamer-isolated CMV-specific $\mathrm{T}$ cells, generated either from their transplant donors $(n=8)$ or from partially HLA matched thirdparty donors $(n=8)$. Better overall response rates were seen in those who received infusions from their own donors with a complete response rate of $64 \% .{ }^{25}$ Pei et al. reported on 32 haplo-identical HSCT recipients with CMV refractory infections, including ten patients with active disease, treated with CMV-specific cytokine-induced $\mathrm{T}$ cells from CMV-seropositive healthy donors. A total of $84 \%$ of the patients cleared the infection within one month from the $\mathrm{T}$ cell infusion. No recurrence was reported. Interestingly, the Authors found that the exhausted phenotype of CMV specific T cells of patients suffering from repetitive CMV infections/disease could be abrogated by the adoptive transfer of CMV-specific T cells in responding patients. ${ }^{26} \mathrm{Blyth}$ et al. treated 30 allogeneic HCT patients with persistent/recurrent viral infections. Mostly due to CMV ( $n=28)$ with partially HLA-matched third-party ex vivo expanded virus-specific T cells. Complete viral clearance was achieved in $76 \%$ without product-related infusion toxicity or de novo GVHD onset. Viral control persisted more than 100 days in all responders. Antiviral therapy was re-introduced in 5 patients. Increase of CD8+ effector T-cells was associated with immune-control of viral infections. In responders, the control of viral replication was not associated with persisting CMV-specific T cells from third-party donors, whose DNA was not detectable beyond day 27 from the infusion. The Authors speculated an indirect putative role of third-party virus-specific T cells which may enhance recovery of HSCT donor-derived virus-specificimmunty. ${ }^{27}$

Many clinical trials are actively recruiting patients to test the efficacy of adoptive virusspecific (both CMV and multi-virus) T-cell therapy in the setting of allo-HSCT or solid organ transplants (NCT02982902 - NCT01646645 - NCT02210078 - NCT02532452 - NCT02136797 - 
NCT03067155 - NCT02702427). At the moment, there are no published results from randomized clinical trials evaluating CMV-directed $\mathrm{T}$ cell adoptive therapy.Interestingly, recent reports showed that strain-specific antibodies exerted anti-CMV reactivation activity and that the active transfer of these antibodies intoHSCT recipients is effective in preventing virus reactivation/disease in murine preclinical models. ${ }^{47}$ This fascinating observation that humoral immunity holds a relevant role in the complex process of CMV reactivation after transplant may be a potential fertile seed for new unexpected therapeutic applications.

\section{Expert commentary}

Despite recent advances, management of CMV in allo-HSCT recipients remains an unmet need. With novel therapeutic and diagnostic strategies, the clinical approach to CMV reactivation/infection and disease is rapidly evolving. The role of novel agents, such as LTM in particular, may highly contribute to design a new therapeutically paradigm that shifts from preemptive approaches to more effective prophylaxis schedules. Toxicity profiles and efficacy of new anti-CMV agents should be compared with that of current "standard-of-care" antiviral drugs. Donor-derived or third-party derived CMV specific $\mathrm{T}$ cells transferred after allo-HSCT could contribute to reduce the impact of CMV infection/disease on clinical outcomes. Moreover, the emerging pivotal roles of both cell-mediated immunologic monitoring and virological load testing are clinically evident. In summary, the integration of novel antiviral therapies including CMV specific $\mathrm{T}$ cell therapies, standardized molecular techniques for virological testing and immunologic assays, may be currently combined into one whole strategy to fight a life-threatening post-transplant complication.

\section{Five-year view}

Future guidelines of CMV reactivation/infection treatments may include strategies to define high risk patients during the pre-transplant work up (i.e. baseline CMV serology, type of transplant, donor-recipient HLA compatibility) and tailored-made interventions based on a watchful prospective monitoring of viral replications from blood (and marrow) samples. A more pronounced integration of the kinetics of viral load coupled with specific molecular targets of novel antiviral therapy will guide clinicians at the bedside to individualize treatments that could limit toxicity while sparing antiviral effects. Moreover, the contribution of CMV specific adoptive T-cell therapies may potentially allow shorter courses of antiviral therapy and stimulate host cellular immune response. 


\section{Key Issues}

- Most current antiviral drugs against CMV remains associated with severe side effects and potential drug-resistance despite remarkable process.

- However newer antiviral compounds are emerging as promising agents against CMVresistant strains and may have a role in both the prophylactic/pre-emptive and therapeutic settings.

- Prospective CMV monitoring in the virology lab will be a key factor to guide therapeutic interventions and significantly improve clinical outcomes

- Off-the-shelf third-party CMV specific T-cells alone or in combination with CMV specific drugs with an acceptable toxicity profile may become crucial for the treatment of lifethreatening CMV disease in critically ill patients.

- A wide-spread role of CMV-vaccination remains to be fully determined 


\section{References}

1. Winston, D. J. et al.Ganciclovir prophylaxis of cytomegalovirus infection and disease in allogeneic bone marrow transplant recipients. Results of a placebo-controlled, double-blind trial. Ann. Intern. Med.118, 179-184 (1993).

2. Goodrich, J. M. et al. Ganciclovir prophylaxis to prevent cytomegalovirus disease after allogeneic marrow transplant. Ann. Intern. Med.118, 173-178 (1993).

3. Montesinos, P. et al. Incidence, risk factors, and outcome of cytomegalovirus infection and disease in patients receiving prophylaxis with oral valganciclovir or intravenous ganciclovir after umbilical cord blood transplantation. Biol. Blood Marrow Transplant.15, 730-740 (2009).

4. Ordemann, R. et al. Foscarnet--an alternative for cytomegalovirus prophylaxis after allogeneic stem cell transplantation? Ann. Hematol.79, 432-436 (2000).

5. Ljungman, P. et al. Cidofovir for cytomegalovirus infection and disease in allogeneic stem cell transplant recipients. The Infectious Diseases Working Party of the European Group for Blood and Marrow Transplantation. Blood97, 388-392 (2001).

6. Melendez-Munoz, R. et al. Cytomegalovirus Infection Incidence and Risk Factors Across Diverse Hematopoietic Cell Transplantation Platforms Using a Standardized Monitoring and Treatment Approach: A Comprehensive Evaluation from a Single Institution. Biol. Blood Marrow Transplant. (2018). doi:10.1016/j.bbmt.2018.10.011

7. Jaing, T.-H. et al. Factors associated with cytomegalovirus infection in children undergoing allogeneic hematopoietic stem-cell transplantation. Medicine (Baltimore)98, e14172 (2019).

8. Teira, P. et al. Early cytomegalovirus reactivation remains associated with increased transplantrelated mortality in the current era: a CIBMTR analysis. Blood127, 2427-2438 (2016).

9. Green, M. L. et al. Cytomegalovirus viral load and mortality after haemopoietic stem cell transplantation in the era of pre-emptive therapy: a retrospective cohort study. Lancet Haematol3, e119-127 (2016).

10. Meesing, A. \& Razonable, R. R. New Developments in the Management of Cytomegalovirus Infection After Transplantation. Drugs78, 1085-1103 (2018).

11. Boeckh, M. \& Ljungman, P. How we treat cytomegalovirus in hematopoietic cell transplant recipients. Blood113, 5711-5719 (2009).

12. El Chaer, F., Shah, D. P. \& Chemaly, R. F. How I treat resistant cytomegalovirus infection in hematopoietic cell transplantation recipients. Blood128, 2624-2636 (2016).

13. Yahav, D. et al. Antiviral prophylaxis in haematological patients: systematic review and meta-analysis. Eur. J. Cancer45, 3131-3148 (2009).

14. Lazzarotto, T. et al. Cytomegalovirus and Epstein-Barr Virus DNA Kinetics in Whole Blood and Plasma of Allogeneic Hematopoietic Stem Cell Transplantation Recipients. Biol. Blood Marrow Transplant.24, 1699-1706 (2018).

15. Costa, C. et al. Comparison of two molecular assays for detection of cytomegalovirus DNA in whole blood and plasma samples from transplant recipients. New Microbiol.39, 186-191 (2016).

16. Lilleri, D. et al. Multicenter quality control study for human cytomegalovirus DNAemia quantification. New Microbiol.32, 245-253 (2009).

17. Melendez, D. P. \& Razonable, R. R. Letermovir and inhibitors of the terminase complex: a promising new class of investigational antiviral drugs against human cytomegalovirus. Infect Drug Resist8, 269-277 (2015).

18. Marty, F. M. et al. Letermovir Prophylaxis for Cytomegalovirus in Hematopoietic-Cell Transplantation. N. Engl. J. Med.377, 2433-2444 (2017).

19. Fryer, J. F. et al. Collaborative study to evaluate the proposed 1st [first] WHO international standard for human cytomegalovirus (HCMV) for nucleic acid amplification (NAT)-based assays. (2010). 
20. Boeckh, M. \& Nichols, W. G. The impact of cytomegalovirus serostatus of donor and recipient before hematopoietic stem cell transplantation in the era of antiviral prophylaxis and preemptive therapy. Blood103, 2003-2008 (2004).

21. Schmidt-Hieber, M. et al. CMV serostatus still has an important prognostic impact in de novo acute leukemia patients after allogeneic stem cell transplantation: a report from the Acute Leukemia Working Party of EBMT. Blood122, 3359-3364 (2013).

22. Ljungman, P. et al. Donor cytomegalovirus status influences the outcome of allogeneic stem cell transplant: a study by the European group for blood and marrow transplantation. Clin. Infect. Dis.59, 473-481 (2014).

23. Navarro, D. et al. Efficacy and Safety of a Preemptive Antiviral Therapy Strategy Based on Combined Virological and Immunological Monitoring for Active Cytomegalovirus Infection in Allogeneic Stem Cell Transplant Recipients. Open Forum Infect Dis3, ofw107 (2016).

24. Boeckh, M. et al. Late cytomegalovirus disease and mortality in recipients of allogeneic hematopoietic stem cell transplants: importance of viral load and T-cell immunity. Blood101, 407-414 (2003).

25. Yong, M. K., Lewin, S. R. \& Manuel, O. Immune Monitoring for CMV in Transplantation. Curr Infect Dis Rep20, 4 (2018).

26. Tey, S.-K. et al. Clinical assessment of anti-viral CD8+ T cell immune monitoring using QuantiFERON-CMV® assay to identify high risk allogeneic hematopoietic stem cell transplant patients with CMV infection complications. PLoS ONE8, e74744 (2013).

27. Bono, P. et al. Quantiferon CMV assay in allogenic stem cell transplant patients. J. Clin. Virol.79, 10-11 (2016).

28. Nesher, L. et al. Utility of the Enzyme-Linked Immunospot Interferon- $\gamma$-Release Assay to Predict the Risk of Cytomegalovirus Infection in Hematopoietic Cell Transplant Recipients. $J$. Infect. Dis.213, 1701-1707 (2016).

29. Yong, M. K. et al. Identifying Cytomegalovirus Complications Using the Quantiferon-CMV Assay After Allogeneic Hematopoietic Stem Cell Transplantation. J. Infect. Dis.215, 1684-1694 (2017).

30. Paouri, B. et al. Quantiferon-Cytomegalovirus assay: A potentially useful tool in the evaluation of CMV-specific CD8+ T-cell reconstitution in pediatric hematopoietic stem cell transplant patients. Pediatr Transplant22, e13220 (2018).

31. Manuel, O. et al. Assessment of cytomegalovirus-specific cell-mediated immunity for the prediction of cytomegalovirus disease in high-risk solid-organ transplant recipients: a multicenter cohort study. Clin. Infect. Dis.56, 817-824 (2013).

32. Costa, C. et al. Evaluation of CMV-specific cellular immune response by EliSPOT assay in kidney transplant patients. J. Clin. Virol.61, 523-528 (2014).

33. Kumar, D., Mian, M., Singer, L. \& Humar, A. An Interventional Study Using Cell-Mediated Immunity to Personalize Therapy for Cytomegalovirus Infection After Transplantation. Am. J. Transplant.17, 2468-2473 (2017).

34. De Gracia-Guindo, M. D. C. et al. Cytomegalovirus Infection Monitoring Based on Interferon Gamma Release Assay in Kidney Transplantation. Transplant. Proc.50, 578-580 (2018).

35. Gliga, S. et al. T-Track-CMV and QuantiFERON-CMV assays for prediction of protection from CMV reactivation in kidney transplant recipients. J. Clin. Virol.105, 91-96 (2018).

36. Chiereghin, A. et al. Monitoring of Cytomegalovirus (CMV)-Specific Cell-Mediated Immunity in Heart Transplant Recipients: Clinical Utility of the QuantiFERON-CMV Assay for Management of Posttransplant CMV Infection. J. Clin. Microbiol.56, (2018).

37. Deborska-Materkowska, D. et al. Diagnostic utility of monitoring cytomegalovirus-specific immunity by QuantiFERON-cytomegalovirus assay in kidney transplant recipients. BMC Infect. Dis.18, 179 (2018). 
38. Chemaly, R. F. et al. Letermovir for cytomegalovirus prophylaxis in hematopoietic-cell transplantation. N. Engl. J. Med.370, 1781-1789 (2014).

39. Marty, F. M. et al. A Randomized, Double-Blind, Placebo-Controlled Phase 3 Trial of Oral Brincidofovir for Cytomegalovirus Prophylaxis in Allogeneic Hematopoietic Cell Transplantation. Biol. Blood Marrow Transplant.25, 369-381 (2019).

40. Marty, F. M. et al. Maribavir prophylaxis for prevention of cytomegalovirus disease in recipients of allogeneic stem-cell transplants: a phase 3, double-blind, placebo-controlled, randomised trial. Lancet Infect Dis11, 284-292 (2011).

41. Papanicolaou, G. A. et al. Maribavir for Refractory or Resistant Cytomegalovirus Infections in Hematopoietic-cell or Solid-organ Transplant Recipients: A Randomized, Dose-ranging, Double-blind, Phase 2 Study. Clin. Infect. Dis. (2018). doi:10.1093/cid/ciy706

42. Nakamura, R. et al.Viraemia, immunogenicity, and survival outcomes of cytomegalovirus chimeric epitope vaccine supplemented with PF03512676 (CMVPepVax) in allogeneic haemopoietic stem-cell transplantation: randomised phase 1b trial. Lancet Haematol3, e87-98 (2016).

43. La Rosa, C. et al. Rapid Acquisition of Cytomegalovirus-Specific $\mathrm{T}$ Cells with a Differentiated Phenotype, in Nonviremic Hematopoietic Stem Transplant Recipients Vaccinated with CMVPepVax. Biol. Blood Marrow Transplant. (2018). doi:10.1016/j.bbmt.2018.12.070

44. La Rosa, C. et al. MVA vaccine encoding CMV antigens safely induces durable expansion of CMV-specific T cells in healthy adults. Blood129, 114-125 (2017).

45. Riddell, S. R. et al. Restoration of viral immunity in immunodeficient humans by the adoptive transfer of T cell clones. Science257, 238-241 (1992).

46. Leen, A. M. et al. Multicenter study of banked third-party virus-specific T cells to treat severe viral infections after hematopoietic stem cell transplantation. Blood121, 5113-5123 (2013).

47. Martins, J. P. et al. Strain-specific antibody therapy prevents cytomegalovirus reactivation after transplantation. Science363, 288-293 (2019). 